\title{
A quantitative comparison of articles on two pests and two pathogens of biosecurity risk to New Zealand extracted from International and Chinese databases
}

\author{
B. Xu ${ }^{1}$, N. Shaw ${ }^{1}$, M. Gee ${ }^{1}$ and D.A.J. Teulon ${ }^{1,2,3}$ \\ ${ }^{1}$ The New Zealand Institute for Plant \& Food Research Ltd, Private Bag 4704, Christchurch, \\ 8140, New Zealand \\ ${ }^{2}$ Better Border Biosecurity, New Zealand (B3nz.org) \\ ${ }^{3}$ Corresponding author: David.Teulon@plantandfood.co.nz
}

\begin{abstract}
New Zealand's increasing trade and tourism with Asia, and especially China, brings with it the need to understand the biosecurity risk to New Zealand from these countries. This study compared the number of articles on two Chinese horticultural pests and two Chinese horticultural plant pathogens, both of biosecurity relevance for New Zealand, which were extracted using web-based searches conducted on two international and three Chinese databases. There were up to 35 times more articles found in Chinese databases searched with Chinese characters than found in international databases searched with Latin script. The quality of information from the articles in any of these databases was not examined. Based on these results, it would be prudent to explore further the information found in Chinese articles stored in Chinese databases to determine their relevance to New Zealand's biosecurity system. Searching Chinese databases with Chinese characters, in combination with the more usual searches in international databases, might ensure a more comprehensive coverage and could help to reduce the biosecurity risk to New Zealand in the future.
\end{abstract}

Keywords: invasive species, biosecurity risk, article retrieval, China, database evaluation, horticultural pest, horticultural pathogen

\section{INTRODUCTION}

Knowledge of the plant pest and disease status of species not established in New Zealand is critical for assessing their potential to compromise our biosecurity. New Zealand agro-ecosystems are dominated by invasive pests and diseases that have mostly originated from elsewhere. New species continue to threaten New Zealand's border, with some successfully establishing each year (Goldson et al. 2015). A strong and effective biosecurity system at the border protects New Zealand from invasive pests that can be very costly to control when eradication is impossible. Invasive pests disrupt market access, leading to significant short and long term financial losses (Nimmo-Bell 2009, Goldson et al. 2015). Historically, much of the information on these invasive species has been relatively easily sourced from countries that were New Zealand's predominant trading partners and 
Table 1 Search entries made in Chinese and international databases

\begin{tabular}{lll}
\hline & Main search topic (example only) & Qualifier \\
\hline Scopus & Article Title, Abstract, Keywords = 'Delia antiqua' & All fields = 'China' or 'Chinese' \\
WoS & Topic = 'Delia antiqua' & Address = 'China' or 'Chinese' \\
WoS+ & Topic = 'Delia antiqua' and '葱蝇' & Address = 'China' or 'Chinese' \\
CNKI & Subject = 'Delia antiqua' and '葱蝇' & 'Precise search' \\
VIP & Article Title, Keywords = 'Delia antiqua' and '葱蝇” & - \\
Wanfang & Subject = 'Delia antiqua' and '葱蝇' & 'Precise search' \\
\hline
\end{tabular}

WoS $=$ Web of Science, WoS $+=$ Web of Science including Chinese Science Citation Index, CNKI = Chinese National Knowledge Infrastructure, VIP = Chongqing VIP Information, Wanfang = Wanfang Data (see Appendix for details). Chinese characters used for species: Delia antiqua (葱蝇), Contrarinia pyrivora (梨瘦蚊), Gymnosporangium spp. (梨锈病), Coniella diplodiella (葡萄白腐病)

from which many tourists originated (e.g. Australia, Europe, North America, the Pacific Islands). These information sources (e.g. written correspondence, internet sites, conference presentations, databases) were in well-understood languages and were relatively accessible. However, New Zealand's recent growing diversification of trade and tourism, especially with Asia, where different languages, science traditions and biosecurity resources are found, adds an additional degree of complexity for New Zealand's biosecurity system. A country of increasing biosecurity concern for New Zealand is China, which is now New Zealand's top trading partner (Statist ics New Zealand 2015) and the source of the second largest group of tourists entering New Zealand (Tourism New Zealand, 2015). China is a very large Asian country using a non-Latin based language script (logograms) and with an increasing volume of published information on agriculture and biological sciences (including biosecurity-related literature). In these disciplines, China was ranked 17 th for published journal articles in 1996 but second (behind the United States) by 2014 (SCImago Journal and Country Rankings 2015 (SJR)). Thus, greater access to Chinese-language information (in both English and Chinese) has the potential to strengthen New Zealand's biosecurity system through increased awareness of pest and disease threats from China.
This study compared the number of articles found in web-based searches of two Chinese horticultural pests and two Chinese horticultural plant pathogens, which are both of biosecurity significance for New Zealand, extracted from two international databases (generally in English) and three Chinese databases (generally in Chinese). Latin script and Chinese characters were used as search subjects. The quality of the articles was not examined but we acknowledge that this is an important additional step in fully understanding the implications of the results presented here.

\section{METHODS \\ Databases}

Web of Science (WoS) and Scopus were chosen as the international databases, as they are commonly used by New Zealand researchers to extract articles for biosecurity information (N. Shaw and M. Gee, Plant \& Food Research Ltd, personal observation). The WoS version, currently licensed to Plant \& Food Research, as well as an expanded version of WoS (WoS+) that includes the Chinese Science Citation Database (CSCD) (CSCD 1997), were used. Three Chinese databases that are considered the major digital literature sources for scientific research in China (Wang \& Wu 2008; Cohen et al. 2015) were also selected: Chinese National Knowledge Infrastructure (CNKI) (accessed 
from their international website as opposed to their national website) (CNKI 1996), Chongqing VIP Information (VIP) (VIP 1989), and Wanfang Data (Wanfang) (Wanfang 1999). The features of these databases are summarised in the Appendix.

\section{Pests and pathogens}

Two horticultural pests, Delia antiqua (Meigen 1826) or onion fly, and Contarinia pyrivora (Riley 1886) or pear midge; and two horticultural pathogens, Gymnosporangium spp. or pear rust disease, and Coniella diplodiella (Speg.) Petr. \& Syd., (1927) or grapevine white rot disease, were selected for the database comparisons. These species were identified in recent Import Risk Analyses as biosecurity concerns to New Zealand (Biosecurity NZ 2009abc) from garlic, pears, and table grapes, as these were all crops under consideration for importation from China at that time. These particular species were chosen because they had only one or two main host plants, thereby simplifying the search strategy as there was no need to specify the crop as well. There are multiple Gymnosporangium species which can cause pear rust disease (Ziller 1961; Dong et al. 2006; Zheng et al. 2013); however, only Gymnosporangium fuscum DC. 1805 was considered as a hazard species in Biosecurity NZ's pear import risk analysis (Biosecurity NZ 2009b). We searched Gymnosporangium species in general (with both Latin script and Chinese characters - see below), but considered only articles that were related to pear rust disease in China in our comparisons.

\section{Database searching and data evaluation}

All databases were searched for the selected organism's scientific names using Latin script and WoS+ and Chinese databases were searched for scientific names in Chinese characters. Simplified Chinese characters of the official written language of mainland China were used (Chu et al. 2012). Chinese characters for each pest and pathogen were determined by an initial search for scientific names in the Chinese databases and then by matching them to appropriate Chinese characters for each organism. This was repeated three to four times to ensure an accurate translation had been achieved (Table 1).

For each database, we chose the option that allowed us to search all the sub-databases (or featured databases - see Appendix) simultaneously. Search strategies were kept as simple as possible, using scientific names without search restrictions or qualifiers, to ensure the search retrieved all the information which might be of potential biosecurity interest. The 'Precise Search' qualifier found in both CNKI and Wanfang databases was used to focus the search on the targeted species. This feature was not available in the version of the VIP database we used. Additionally, the searches in the international databases were restricted to "China or Chinese" to ensure the search results were related to China. As this restriction was not available in the Chinese databases, all Chinese articles (either title and/or abstract) were reviewed to ensure that the citation referred to the pest species in China and not elsewhere. This review also helped to remove articles which were not relevant to the target species. Comparisons with the international databases were made on this 'reviewed' set of articles.

The evaluation of the different databases in relation to the relevant biosecurity articles they extracted was based on a comparison of the number of articles found in each database for each pest and disease species. The results were contrasted for two different time periods: firstly, all citations found in a given database (Appendix), and secondly, only articles published between 1 January 2010 and 31 December 2015, to provide a more equivalent comparison. The degree of overlapping articles (i.e. duplicates) in Latin script and Chinese characters searches (combined) from one international (WoS+) and one Chinese (CNKI) database was analysed to explore differences between databases further. As is standard practice for a survey, data have been summarised using tabulation and summary statistics. In terms of the Preferred Reporting Items for Systematic Review and Meta-Analysis (PRISMA) (PRISMA 
2009), our study covered 'identification' and 'screening', partly 'eligibility' but not 'included' (i.e. not the quantitative synthesis). The quality of the articles was not examined because of resource constraints, but this is acknowledged as an important additional step in fully understanding the implications of the results presented here.

\section{RESULTS}

With one exception (Scopus/Contarinia pyrivora), all international and Chinese databases searched with Latin script and Chinese characters found articles for the two Chinese pests and two Chinese pathogens of biosecurity interest to New Zealand.

\section{Latin script searches}

In general, the number of articles found from Latin script (i.e. scientific name) searches was similar for both international and Chinese databases, except for CNKI, which had the highest numbers of articles for three species; and for Scopus, which had the lowest number of articles for all four species. For CNKI, approximately twice as many articles were found for two species (Delia antiqua, Gymnosporangium spp.) as in other databases excluding Scopus. For Scopus, results ranged from as low as $10 \%$ fewer articles than found in some other databases (Table 2). These results were similar for the restricted search period (2010-2015).

\section{Chinese character searches}

Far fewer articles were found in WoS+ than in Chinese databases with Chinese character searches. CNKI and VIP had similar numbers of articles and both had slightly more than Wanfang. These results were similar for the restricted search period (2010-2015).

\section{Latin script vs Chinese character searches}

Two to ten times more articles were found in all the Chinese databases for all species using Chinese characters rather than Latin script searches (Table 2). For WoS+, more articles were found for the two species using Latin script searches; more articles were found for one species using Chinese character searches; and the same number of articles was found the fourth species by using the different search methods. Of note is that the number of articles found for Coniella diplodiella nearly doubled when using Chinese character-only searches in WoS+ compared with Latin script-only searches in WoS. Duplicates of articles from Latin script and Chinese character searches were not analysed in the WoS+ or the Chinese databases, so the results may overestimate differences in searches between Latin script and Chinese character searches. The results were similar for the restricted search period (2010-2015).

\section{International vs Chinese database searches}

For all pest and disease species, searches in the Chinese databases yielded many more articles than the international databases (Table 2). More articles were found in the Chinese databases searched using Chinese script only than in the WoS, searched in Latin script only. Clearly differences between Scopus and Chinese databases were greater because fewer articles were found in Scopus than in WoS. The increased number of articles found in Chinese databases compared with WoS were: Delia antiqua - CNKI (x6), VIP (x3), Wanfang (x2); Contarinia pyrivora - CNKI (x14), VIP (x14), Wanfang (x10); Gymnosporangium spp. - CNKI (x8), VIP (x5), Wanfang (x4); and Coniella diplodiella - CNKI (x19), VIP (x17), Wanfang (x11). If articles from Chinese character searches in WoS+ were included in the comparison, the increase would be smaller by a factor of about half. However, if Latin searches in Chinese databases were also included, the difference might be greater depending on the degree of overlap between Latin script and Chinese character searches in these databases (duplicates between Latin script and Chinese characters were not analysed in the Chinese databases). Interestingly, the difference between WoS and Chinese databases generally increased for the restricted search period (2010-2015). Increased 


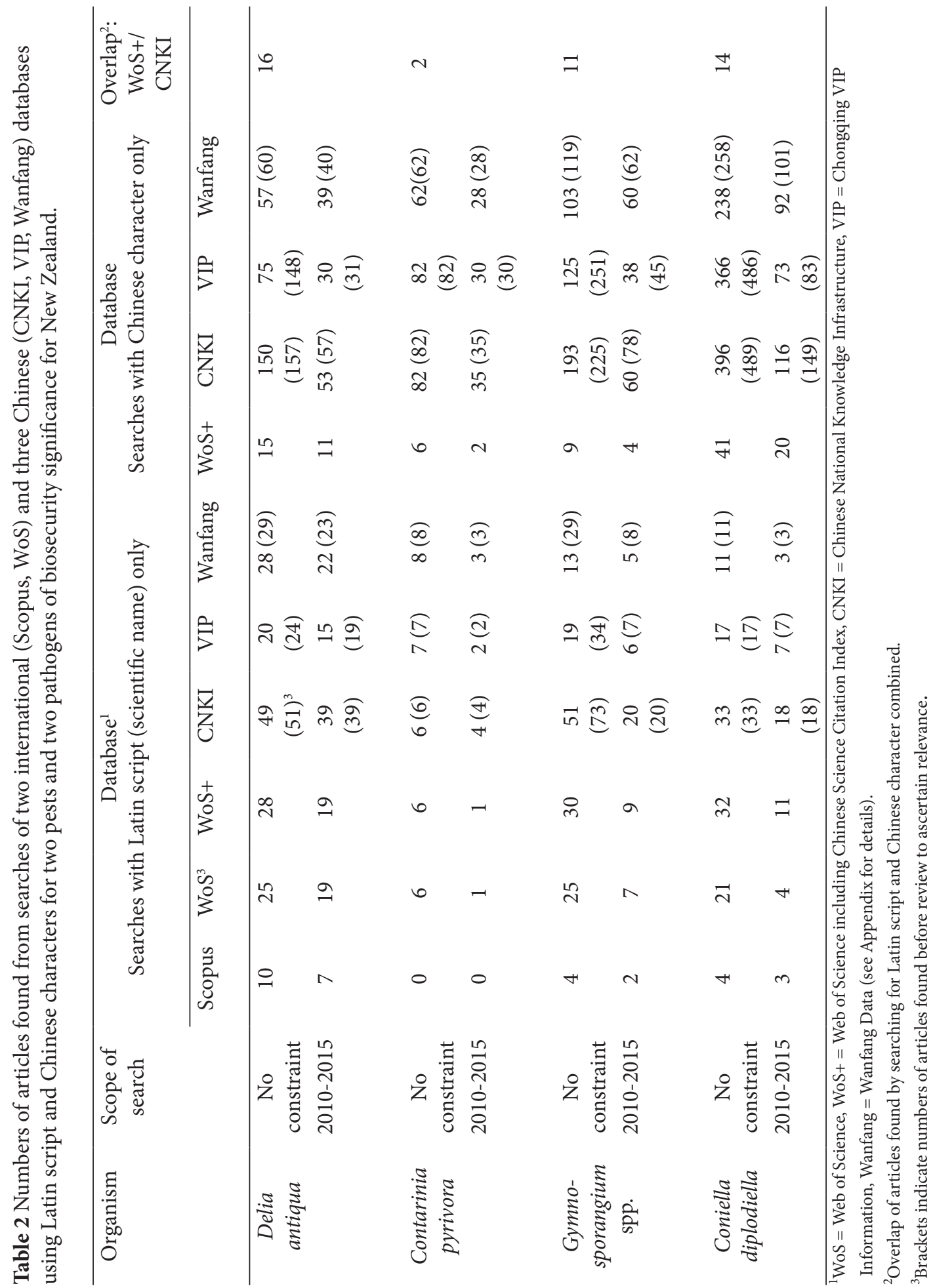


numbers of articles compared with those in WoS for this time period were: Delia antiqua CNKI (x3), VIP (x2), Wanfang (x2); Contarinia pyrivora - CNKI (x35), VIP (x30), Wanfang (x28); Gymnosporangium spp. - CNKI (x9), VIP (x5), Wanfang (x9); and Coniella diplodiella - CNKI (x29), VIP (x18), Wanfang (x23). It is worth noting that the CNKI international database which was used in this study does not include access to international journals whereas the CNKI national database (available within China) does.

\section{Overlap between WoS+ and CNKI}

There was some degree of overlap of articles found by searching in CNKI and WoS+ (for both combined Latin script and Chinese character searches). Between 16 and 38\% of articles found in $\mathrm{WoS}+$ were also found in CNKI. However, most papers published in non-Chinese journals found in WoS+ were not found in CNKI.

\section{Review}

Manually reviewing titles and/or abstracts from articles found in the three Chinese databases (Latin script and Chinese character searches) confirmed that the majority (>90\%) of articles were about pest and pathogen species in China. However, for Gymnosporangium spp. and Coniella diplodiella, this value was generally lower (between 75-93\%). On two occasions (VIP/Gymnosporangium spp. and $\mathrm{VIP} /$ Delia antiqua), this value was closer to $50 \%$.

\section{DISCUSSION}

The aim of this study was to compare the number of articles found in web-based searches for two Chinese horticultural pests and two Chinese pathogens of biosecurity relevance for New Zealand from international databases and Chinese databases, using Latin script and Chinese characters as search subjects. While the CNKI database produced a moderate increase in articles with Latin script searches compared with the international databases, the most noteworthy difference was the large increase in articles found in Chinese databases searched with Chinese characters (up to x35 increase) (Table 2).

Searches (Latin script and Chinese character searches combined) for the two insect pests and two plant pathogens in international and Chinese databases found the following trend in terms of numbers of articles: Scopus $<$ WoS $<$ WoS+ $<$ Wanfang $<$ VIP $<$ CNKI (Table 2). This trend was consistent across the four species, but for the constrained search (2010-2015) fewer articles were found in VIP than in Wanfang. All three Chinese databases are much younger than the international databases (Appendix), so this trend could not be accounted for by database age. Interestingly, a similar trend (articles from international vs Chinese databases) occurred for the constrained time period (2010-2015) search, suggesting that this trend is becoming more pronounced over time. The overlap of articles between both WoS+ and CNKI was relatively small, with WoS+ tending to find more articles published by Chinese researchers in international journals (mostly in English) whereas the international CNKI predominantly sourced Chinese journals with a strong focus on issues within China.

Clearly, the quality of information found in articles will be of paramount importance to New Zealand's border biosecurity, but this was beyond the scope of this project partly because of the large number of articles found in the Chinese database searches. The titles and/or abstracts of all articles resulting from Chinese database searches were reviewed briefly to ensure that these articles were relevant to the two pests and two plant pathogens and were found in China, but otherwise there was no attempt to measure the quality of any articles in this study.

Detailed descriptions of CNKI, VIP and Wanfang are found in the Appendix. These Chinese databases are considered the major digital literature sources for scientific research in China (Wang \& Wu 2008; Cohen et al. 2015). However, it may be useful to identify and analyse additional databases to see if the trends found in this study have wider application. Google 
Scholar could be considered as a useful source of information for both Latin script and Chinese characters, but it is currently blocked for use in China (Stapleton 2015), which may limit its current utility. Furthermore, it may be that certain databases provide more informative (as in "fit for purpose") articles for particular areas of interest (e.g. pest management) than other databases and this could be explored. Different databases have different options for refining the research strategy. For example, the 'Precise Search' was not available for the version of VIP used here and many articles found were not as relevant (Table 2).

To fully exploit the biosecurity information found in Chinese databases, a knowledge of the Chinese language is clearly beneficial, if not mandatory. Initially, accurate translation of Latin scientific names to Chinese names in Chinese characters is required. This issue was made more complicated where scientific names were not available in Chinese databases, or where more than one Latin named species used identical Chinese names (e.g. Contarinia pyrivora and Dasyneura pyri; different Gymnosporangium species). In these instances we relied on our brief review to eliminate irrelevant articles. While Chinese databases predominantly collect journals in Chinese (Appendix), some also provide English titles and abstracts. Lack of fluency in Chinese understandably restricts the interpretation of an article. Automation of scientific translations from English to Chinese are still considered to be suboptimal; a Google Translate study found that Chinese to English had the least accurate translation rate (8-30\%) (Balk et al. 2012).

Overall, this study suggests that optimal and effective searching strategies for information on pest and disease species in China necessitates searching Chinese databases with Chinese characters in combination with the more usual searches in international databases, to ensure comprehensive coverage.

\section{ACKNOWLEDGEMENTS}

Simon Bulman and Max Suckling (both Plant \& Food Research) provided very useful critiques of an initial draft of this study. This study was funded through a New Zealand Institute for Plant \& Food Research Ltd Summer Studentship to Bingqin (Grace) $\mathrm{Xu}$, aligned to the Better Border Biosecurity research collaboration.

\section{REFERENCES}

Balk EM, Chung M, Hadar N, Patel K, Yu WW, Trikalinos TA, Chang LKW 2012. Accuracy of Data Extraction of Non-English Language Trials with Google Translate [Internet]. In: Methods Research Reports 12-EHC056EF. Rockville (MD), Agency for Healthcare Research and Quality (US).

Biosecurity New Zealand 2009a. Import Risk Analysis: Onion (Allium cepa Liliaceae) fresh bulbs for consumption from China. MAF Biosecurity, Wellington, New Zealand. 275 p.

Biosecurity New Zealand 2009b. Import Risk Analysis: Pears (Pyrus bretschneideri, Pyrus pyrifolia, and Pyrus sp. nr. communis) fresh fruit from China: Final. MAF Biosecurity, Wellington, New Zealand. 454 p.

Biosecurity New Zealand 2009c. Import Risk Analysis: Table grapes (Vitis vinifera) from China: Final. MAF Biosecurity, Wellington, New Zealand. 314 p.

Chu C, Nakazawa T, Kurohashi S 2012. Chinese Characters Mapping Table of Japanese, Traditional Chinese and Simplified Chinese. LREC: 2149-2152.

CNKI 1996. China Knowledge Resource Integrated Database. http://oversea.cnki.net/ (accessed January 2016).

Cohen JF, Korevaar DA, Wang J, Spijker R, Bossuyt PM 2015. Should we search Chinese biomedical databases when performing systematic reviews? Systematic reviews 4: 23.

CSCD 1997. Chinese Science Citation Database. http://sciencechina.cn/index_more1.jsp (accessed January 2016).

Dong XL, Li BH, Zhang ZF, Li BD, Xu XM 2006. Effect of environmental conditions on germination and survival of teliospores and basidiospores of the pear rust fungus (Gymnosporangium asiaticum). European 
Journal of Plant Pathology 115: 341-350.

Falagas ME, Pitsouni EI, Malietzis GA, Pappas G 2008. Comparison of PubMed, Scopus, web of science, and Google scholar: strengths and weaknesses. The FASEB journal 22: 338-342.

Goldson SL, Bourdôt GW, Brockerhoff EG, Byrom AE, Clout MN, McGlone MS, Nelson WA, Popay AJ, Suckling DM, Templeton MD 2015. New Zealand pest management: current and future challenges, Journal of the Royal Society of New Zealand DOI: 10.1080/03036758.2014.1000343.

Nimmo-Bell 2009. Economic costs of pests to new Zealand. MAF Biosecurity, Wellington, New Zealand. 80 p.

PRISMA 2005. PRISMA 2009 flow diagram. http://prisma-statement.org/documents/ PRISMA\%202009\%20flow\%20diagram.pdf (accessed April 2016).

SCImago 2015. SCImago Journal and Country Rankings. http://www.scimagojr.com/ countryrank.php (accessed January 2016).

Stapleton P 2015. China must unblock Google Scholar and keep the internet open for its researchers. http://www.scmp.com/ comment/insight-opinion/article/1883500/ china-must-unblock-google-scholar-andkeep-internet-open-its researchers (accessed April 2016).

Statistics New Zealand 2015. Global New Zealand - International trade, investment, and travel profile: year ended December 2014. http://www.stats.govt.nz/browse for stats/ industry sectors/imports and exports/ global-nz-dec-14/key-points.aspx (accessed January 2016).

Tourism New Zealand 2015. Markets and Stats. http://www.tourismnewzealand.com/ markets-stats/ (accessed January 2016).

VIP 1989. VIP Journal Integration platform VJIP. http://lib.cqvip.com/ (accessed January 2016).

Wanfang 1999. Cross database search. http:// www.wanfangdata.com/db search.asp (accessed January 2016). 王平南, 吴娅娜 (Wang P, Wu Y) 2008. 国内三大期刊全文数 据库优势与特色评述 (Comparison of three key Chinese digital databases: strengths and weaknesses). 图书馆(5): 88-89.

Zheng Y, Duan X, Cui X, Sun Y, Yang X 2013. Spatial distribution pattern and sampling method of pear rust spot on leaves. Journal of Northeast Forestry University 41: 80-83.

Ziller W 1961. Pear rust (Gymnosporangium fuscum) in North America. Plant Disease Reporter 45(2): 90-94. 




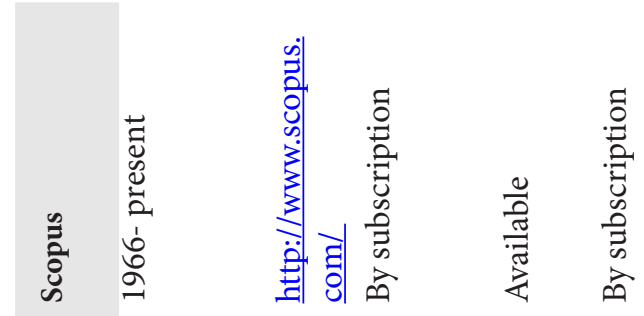
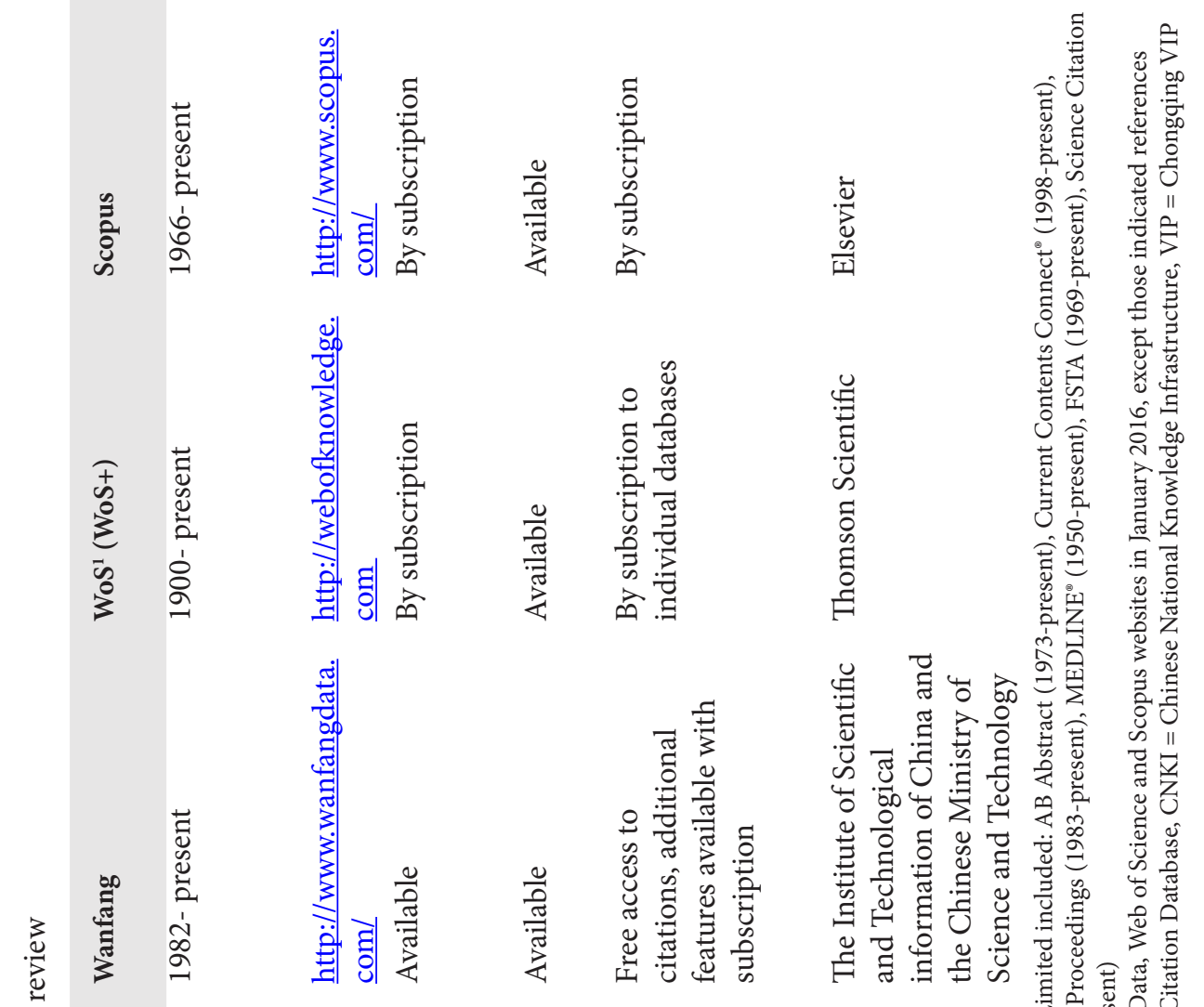

๙ิڤ

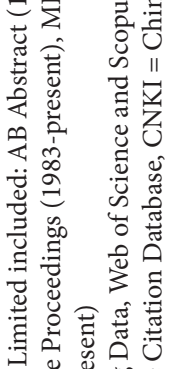

西
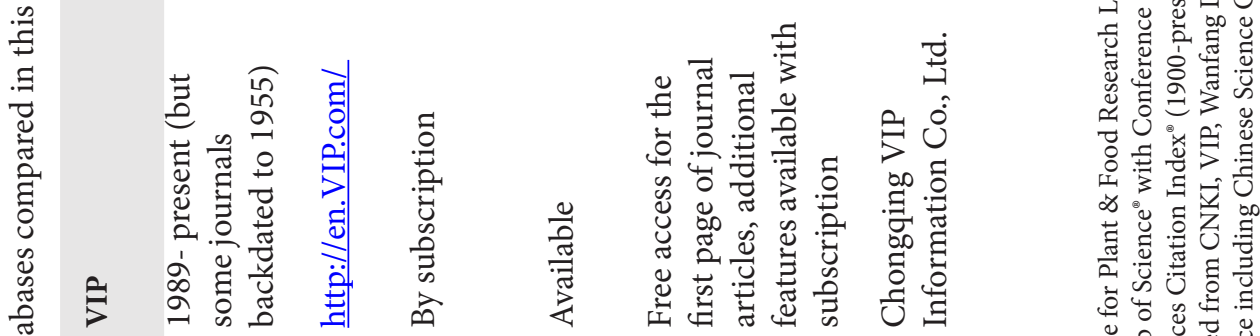

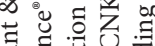

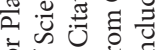

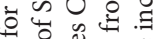

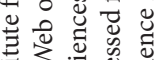

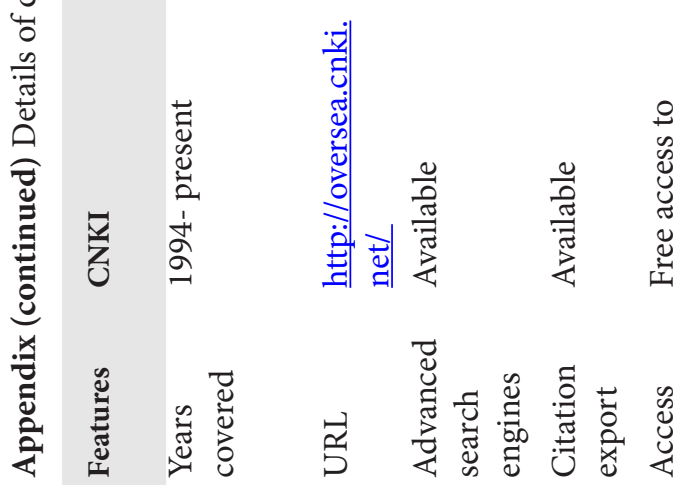

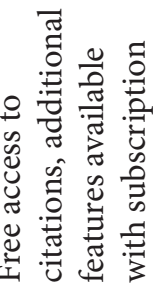

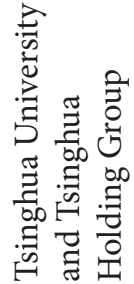

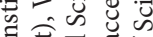

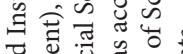

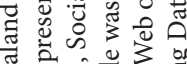

过产言了

उ०

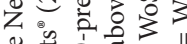
\& ํㅡㄹ च 웜월

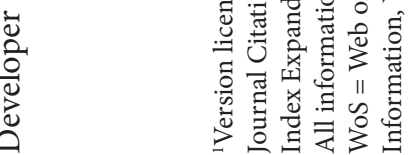

